\title{
Propagation Properties of Airy Beam through Periodic Slab System with Negative Index Materials
}

\author{
Long Jin (iD) and Xingqiang Zhang \\ Department of Basic Science, Hubei University of Automotive Technology, Shiyan 442002, China \\ Correspondence should be addressed to Long Jin; crazyjinlong@163.com
}

Received 23 February 2017; Revised 21 May 2017; Accepted 11 June 2017; Published 16 January 2018

Academic Editor: Sulaiman Wadi Harun

Copyright (c) 2018 Long Jin and Xingqiang Zhang. This is an open access article distributed under the Creative Commons Attribution License, which permits unrestricted use, distribution, and reproduction in any medium, provided the original work is properly cited.

\begin{abstract}
Based on light transfer matrix and electric field vector equation, the evolution of Airy beam propagating in periodic slab system with three negative index materials (NIMs) and its transmission mechanism are investigated. The intensity profiles on emergent surface of periodic slab system and side view of Airy beam propagating in each right handed material (RHM) and double negative material (DNM) unit including lossless and losses DNMs are discussed. It is revealed that the self-recovery Airy beam can be achieved in long distance by using lossless periodic slab system as long as the negative refractive index $n_{l}=-n_{r}$ and each unit length $L=Z$. As to losses slab system contained DNMs, the smaller the collision frequencies are, the better the Airy beam quality is formed. It is expected that the proposed manner of beam transmission and corresponding conclusions can be useful for extension applications of optical control, especially for optical communication and optical encryption technique.
\end{abstract}

\section{Introduction}

Airy beam, which makes up one of the important nondiffracting waves, was first described by Berry and Balazs in 1979 by solving Airy solution of the paraxial wave equation [1]. Recently, Siviloglou et al. observed the 1D and 2D Airy phenomena through experiment in 2007 [2]; they utilized spatial light modulator combined with Fourier transform technique to generate the accelerating finite energy Airy beam. From then on, the particular properties of this beam, approximate nondiffraction, parabolic path of the central lobe, self-accelerating in the transverse, and self-recovery, have been an attractive topic for both theoretical and applied study in modern optics [3]. At the same time, a considerable number of frontier and interdisciplinary applications, such as optical microparticles manipulation [4], formation of Airy beam lasers [5], generation of optical bullets [6], routing of surface plasmon polaritons [7], generation of curved plasma channels [8], and creating curved electron beams without any external field [9], have been took advantage of the Airy function and Airy beam technology.

As far as the width and depth are concerned, optical film and related technology had been significantly developed with the progress of science and technology. The preparation process of optical film is using different refractive index materials to coat a periodic media structure [10]. These optical components possessing the properties of spectral transmittance, spectral reflection, spectral absorption, and altering the state of polarization and/or phase of light are widely used in manufacturing high reflectance film, antireflective film, interference filter, and so on [11]. Meanwhile, metamaterials so-called negative index materials (NIMs) have mushroomed in many aspects of material physics and chemistry science [12]. Double negative materials (DNMs) with both permittivity $\varepsilon$ and permeability $\mu$ are known as left handed materials (LHMs), whose extraordinary electromagnetic (EM) properties were first presented by Veselago [13]. Beyond that, there are single negative materials (SNMs) among which only one of the EM parameters is negative; these materials include the mu negative media (MNGM, $\varepsilon>0, \mu<0)$ and the epsilon negative media (ENGM, $\varepsilon<0, \mu>0)[14,15]$. There exist abnormal negative refraction effect [16], inverse Cerenkov radiation, reverse Doppler effect [17], and negative radiation pressure [18] in NIMs because the electromagnetic wave group velocity and phase velocity of these materials are oriented in the opposite 


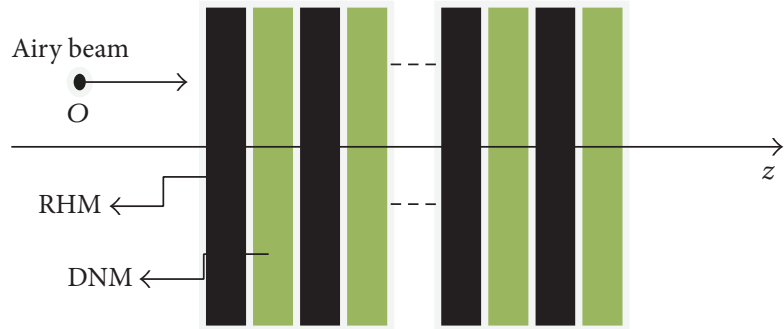

FIGURE 1: Light path schematic diagram of periodic slab system with DNMs.

direction; based on these principles, optical film contained NIMs has broad application prospects in microwave communication, large capacity storage medium, and superresolution imaging.

In the letter, we mainly research the evolution of Airy beam propagation in optical film with DNMs, but what we want to emphasize is that our optical film model is $1 \mathrm{D}$ periodic slab system, with its period number being very small and each unit is measured in metre in order to conveniently observe beam transmission mechanism. The rest of the paper is organized as follows: firstly, the general propagation formulae of the Airy beam propagating through the paraxial optical system are calculated in space domain by transfer matrix and the Collins integral. Furthermore, the intensity profiles on emergent surface of periodic slab system and side view of Airy beam transmission in each RHM and DNM unit including lossless and losses DNMs are discussed. Finally, some important conclusions are summarized. It is expected that the proposed manner of wave transmission and the corresponding conclusions can be useful for extension applications of optical control, especially for optical communication and optical encryption technique.

\section{Physical Model and Formulae}

The schematic diagram of $1 \mathrm{D}$ periodic slab system with NIMs is shown in Figure 1; the medium layer with black colour stands for RHM and the light green is NIM. Here we choose isotropic DNM with its relative permittivity and permeability given by the Drude model [19]:

$$
\begin{aligned}
& \varepsilon_{r}(\omega)=1-\frac{\omega_{p e}^{2}}{\omega\left(\omega+i \gamma_{e}\right)}, \\
& \mu_{r}(\omega)=1-\frac{\omega_{p m}^{2}}{\omega\left(\omega+i \gamma_{m}\right)},
\end{aligned}
$$

where $\omega_{p e}$ and $\omega_{p m}$ are the electric plasma frequency and the magnetic plasma frequency. $\gamma_{e}, \gamma_{m}$ are the electric and magnetic collision frequencies, respectively. The frequency $v=$ $\omega /(2 \pi)$ is measured in $10^{2} \mathrm{THz}$, and $\omega$ is angular frequency. DNM's refractive index $n_{l}=-\sqrt{\varepsilon_{r}(\omega) \cdot \mu_{r}(\omega)}$; the length of each DNM unit is $L$. With regard to RHM, the refractive index and each unit length are $n_{r}$ and $Z$, respectively.
Considering an Airy beam that propagates from $O$ point along the direction of $z$, the electric field at $z=0$ ( $O$ point) can be described as $[20,21]$

$$
\begin{aligned}
E\left(x_{1}, y_{1}\right)= & A i\left(\frac{x_{1}}{x_{0}}\right) \exp \left(\frac{a_{0} x_{1}}{x_{0}}\right) \\
& \times A i\left(\frac{y_{1}}{y_{0}}\right) \exp \left(\frac{b_{0} y_{1}}{y_{0}}\right),
\end{aligned}
$$

where $A i(\cdot)$ denotes the Airy function, $x_{0}$ and $y_{0}$ are beams horizontal size and vertical size, respectively, $a_{0}$ and $b_{0}$ represent exponential modulation factors, and $E(\cdot)$ is electric field intensity on incident surface. The propagation of beam through paraxial periodic slab system with NIMs can be described by the Collins integral [22]:

$$
\begin{gathered}
E_{2}(x, y, z)=\left(\frac{-i}{\lambda B}\right) e^{i k z} \iint_{s_{1}} E_{1}\left(x_{1}, y_{1}, 0\right) \\
\cdot e^{\left((i k / 2 B)\left[A\left(x_{1}^{2}+y_{1}^{2}\right)+D\left(x^{2}+y^{2}\right)-2\left(x_{1} x+y_{1} y\right)\right]\right)} d x_{1} d y_{1},
\end{gathered}
$$

where $\lambda=1.55 \mu \mathrm{m}$ is the wavelength of Airy beam, $k=2 \pi / \lambda$ is the wave number. $A, B, C$, and $D$ are the transfer matrix elements of the total paraxial optical systems $T$ :

$$
T=\left(\begin{array}{ll}
A & B \\
C & D
\end{array}\right) .
$$

Now, let us discuss some useful matrices of optical medium. When beam is traveling $x$ distance in DNM or RHM, the transfer matrix can be written as

$$
M(x)=\left(\begin{array}{ll}
1 & x \\
0 & 1
\end{array}\right) .
$$

While beam propagates from RHM into DNM slab, the transfer matrix form in RHM-DNM interface is given by

$$
M\left(n_{r}, n_{l}\right)=\left(\begin{array}{cc}
1 & 0 \\
0 & \frac{n_{r}}{n_{l}}
\end{array}\right) .
$$

Likewise, DNM-RHM interface transfer matrix is

$$
M\left(n_{l}, n_{r}\right)=\left(\begin{array}{cc}
1 & 0 \\
0 & \frac{n_{l}}{n_{r}}
\end{array}\right) .
$$

By cross-producing these matrices in accordance with the order of Airy beam passing through periodic slab system, we can obtain the general propagation formula of the Airy beam propagating through the paraxial optical systems in the space domain:

$$
\begin{gathered}
E(x)=\frac{1}{\sqrt{A}} A i\left(\frac{x}{A x_{0}}-\frac{B^{2}}{4 A^{2} k^{2} x_{0}^{4}}+i \frac{a_{0} B}{A k x_{0}^{2}}\right) \\
\cdot \exp \left(-i \frac{k C x^{2}}{2 A}\right) \cdot \exp \left(\frac{a_{0} x}{A x_{0}}-a_{0} \frac{B^{2}}{2 A^{2} k^{2} x_{0}^{4}}\right. \\
\left.-i \frac{B^{3}}{12 A^{3} k^{3} x_{0}^{6}}+i \frac{a_{0}^{2} B}{2 A k x_{0}^{2}}+i \frac{B x}{2 A^{2} k x_{0}^{3}}\right),
\end{gathered}
$$




$$
\begin{aligned}
& E(y)=\frac{1}{\sqrt{A}} A i\left(\frac{y}{A y_{0}}-\frac{B^{2}}{4 A^{2} k^{2} y_{0}^{4}}+i \frac{b_{0} B}{A k y_{0}^{2}}\right) \\
& \cdot \exp \left(-i \frac{k C y^{2}}{2 A}\right) \cdot \exp \left(\frac{b_{0} y}{A y_{0}}-b_{0} \frac{B^{2}}{2 A^{2} k^{2} y_{0}^{4}}\right. \\
& \left.\quad-i \frac{B^{3}}{12 A^{3} k^{3} y_{0}^{6}}+i \frac{b_{0}^{2} B}{2 A k y_{0}^{2}}+i \frac{B y}{2 A^{2} k y_{0}^{3}}\right), \\
& E(x, y)=E(x) \times E(y) .
\end{aligned}
$$

And the light intensity is easily obtained by using complex conjugate of $E(x, y, z)$ :

$$
I=\frac{n}{2 c \mu_{0}}|E(x, y, z)|^{2} \propto|E(x, y, z)|^{2} .
$$

\section{Results and Discussion}

To delve into the evolution of Airy beam passes through periodic slab system $(A B)_{m}$, where $A$ and $B$ are RHM and DNM, respectively, and $m=2$ is period number of this system, we carry out Airy beam transmission numerical calculation and display some simulation findings. Here we assume $x_{0}=$ $y_{0}=0.2 \mathrm{~mm}, a_{0}=b_{0}=0.1$, each DNM is lossless, and related parameters are set as $\gamma_{e}=\gamma_{m}=0, v=2 \times$ $10^{2} \mathrm{THz}, \omega_{p e}=\omega_{p m}=2 \pi \nu \times \sqrt{2}, 2 \pi \nu \times \sqrt{2.55}$, and $2 \pi v \times$ $\sqrt{3.1}$, resulting in $n_{l}=-1.00,-1.55$, and -2.10 , respectively, $n_{r}=1.55$ fixed, $L=Z=0.25 \mathrm{~m}$. Firstly, we depict transverse normalized intensity distribution of incident Airy beam and three kinds of emerging beams on output surface. It is easily found that the incident beam distribution and emerging beam transverse profiles are completely the same when DNM's plasma frequencies $\omega_{p e}=\omega_{p m}=2 \pi \nu \times \sqrt{2.55}$ (i.e., $\operatorname{abs}\left(n_{l}\right)=n_{r}=1.55$ ) from Figures $2(\mathrm{a})$ and 2(b), and the normalized central main lobe intensity slightly decreases when $\omega_{p e}=\omega_{p m}=2 \pi v \times \sqrt{3.1}$, which was discerned from the colour bar in Figure 2(c); while $\omega_{p e}=\omega_{p m}=2 \pi v \times$ $\sqrt{2}$ and emerging central maximum intensity is attenuated significantly, the quantity value is 0.57 compared to that of $\omega_{p e}=\omega_{p m}=2 \pi \nu \times \sqrt{2.55}$.

The intensity evolution of Airy beam propagating in periodic slab system $(A B)_{2}$ is deeply investigated in order to show how wave transfers from RHM to DNM; the simulation results are depicted in Figure 3; green arrow lines show the material boundaries; this reveals that the maximum intensity decreases and intensity distribution generates self-bending as the propagation distance increases while the Airy beam propagates in the RHM; when passing through the RHMDNM interface, the beam undergoes negative refraction; in other words, both incident beam and refracted beam are on the same side of the interface normal; subsequently, the beam experiences inverse parabolic trajectory in DNM, which indicates the self-bending in RHM can be compensated and refocused again when wave passes through the DNM. In Figure 3(a), as DNM's $n_{l}=-n_{r}=-1.55$, the transverse pattern could go back to its original feature at 1st end of DNM and operate the stable and excellent beam quality; in consequence, we draw a conclusion that the intensity profiles of incident surface and emergent surface are completely the same on condition that $L=Z$ no matter what the value of period number $m$ is; this case is called Airy beam which can just be compensated by each DNM, and related principle diagram is presented in Figure 3(b). The calculation result in Figure 3(c) reveals that the self-bending in RHM cannot be completely compensated by DNM when $n_{l}=-2.10<-n_{r}$ and $L=Z=$ $2.5 \mathrm{~m}$; this effect accumulates accompanied by increasing of periodic slab system period number $m$ and severely impacts the Airy beam's quality of imaging and transmission. We also depict corresponding principle diagram in Figure 3(d) to describe beam transmission characteristics under this circumstance. Now an interesting question naturally arises: How long is the DNM needed to just compensate self-bending when $n_{l}=-2.10$ ?

When Airy beam passes through the 1st RHM and DNM, the transfer matrix $T$ can be described as

$$
\begin{aligned}
T= & \left(\begin{array}{ll}
A & B \\
C & D
\end{array}\right) \\
= & \left(\begin{array}{ll}
1 & 0 \\
0 & n_{l}
\end{array}\right) \times\left(\begin{array}{ll}
1 & L \\
0 & 1
\end{array}\right) \times\left(\begin{array}{ll}
1 & 0 \\
0 & \frac{n_{r}}{n_{l}}
\end{array}\right) \times\left(\begin{array}{ll}
1 & Z \\
0 & 1
\end{array}\right) \\
& \times\left(\begin{array}{ll}
1 & 0 \\
0 & \frac{1}{n_{r}}
\end{array}\right) .
\end{aligned}
$$

Substituting $Z=2.5 \mathrm{~m}, n_{r}=1.55$, and $n_{l}=-2.10$ into (10), the analytical transfer matrix element $B$ can be formulated as

$$
B=\frac{50}{31}-\frac{10}{21} \times L .
$$

Hence, the necessary distance for just compensating selfbending is $L=105 / 31 \mathrm{~m}$ in DNM. The side intensity evolution of Airy beam propagating in quasiperiodic slab system with $Z=2.5 \mathrm{~m}$ and $L=105 / 31 \mathrm{~m}$ alternately is plotted in Figure 3(e); it is clearly found that the output Airy beam gets back to its original feature at the end of each DNM, which verifies our theoretical analysis. On the contrary, the length of DNM to just compensate self-bending when $n_{l}=$ -1.00 is smaller than $2.5 \mathrm{~m}$. By substituting $Z=2.5 \mathrm{~m}, n_{r}=$ 1.55 , and $n_{l}=-1.00$ into (10), analytical transfer matrix element $B$ can also be written as

$$
B=\frac{50}{31}-L
$$

and the quantity value is $L=50 / 31 \mathrm{~m}$; therefore, the beam intensity self-bends again in the rest of the of 1 st DNM because $L=2.5 \mathrm{~m}$ fixed; when passing through the RHM-DNM interface, the beam undergoes negative refraction as well and then experiences inverse parabolic trajectory even in the next RHM in Figure 3(f); Pendry once explained this overcompensation phenomenon in detail through photoelectromagnetic theory [23], and the principle diagram is depicted in Figure 3(g). 


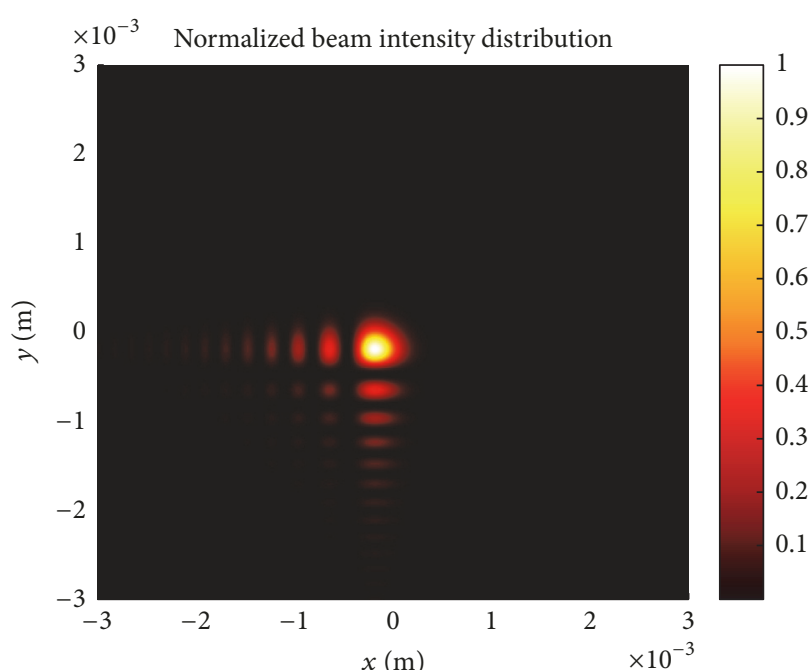

(a)

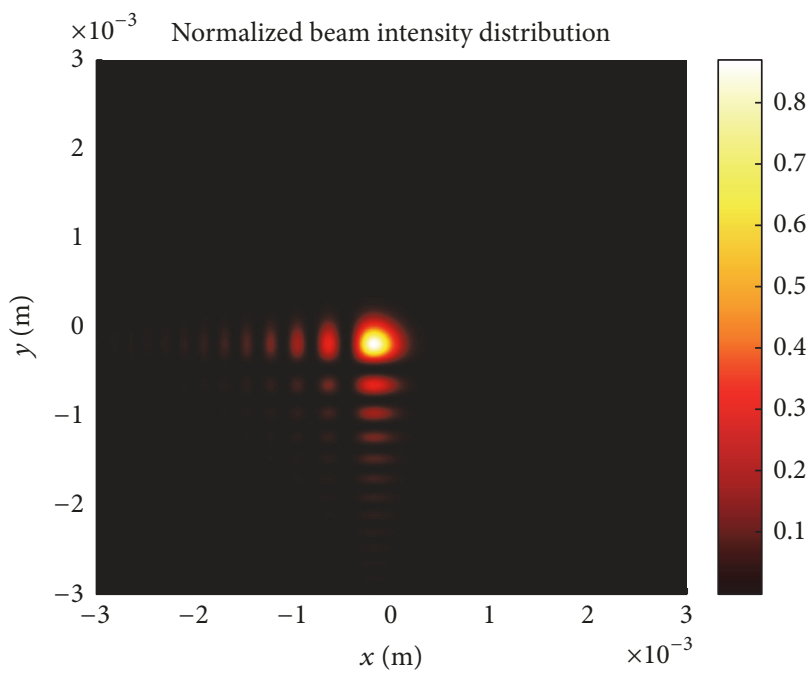

(c)

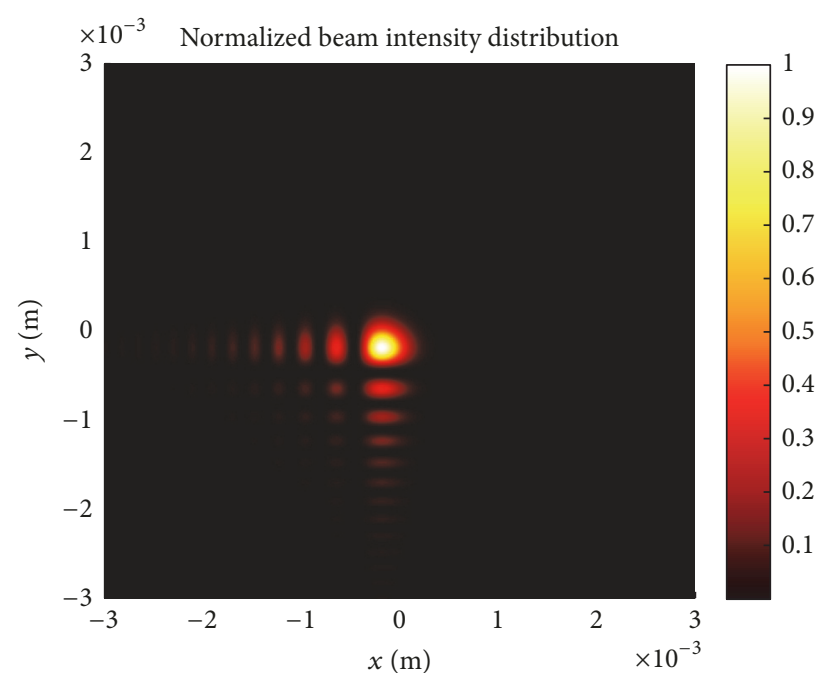

(b)

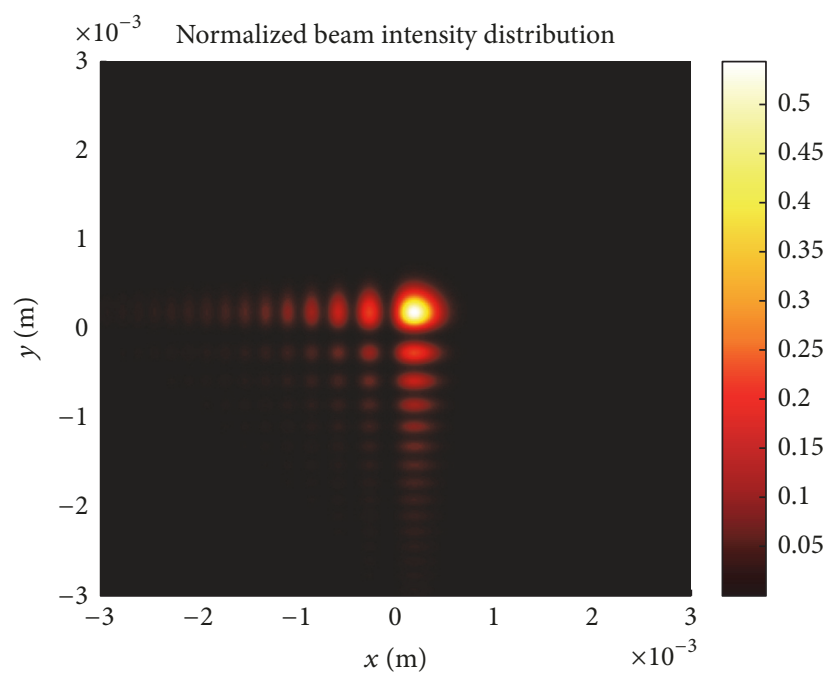

(d)

FIGURE 2: Transverse normalized intensity distribution of incident Airy beam and three kinds of emerging beams on output surface. (a) Incident Airy beam distribution, (b) emerging Airy beam distribution, DNM's $\omega_{p e}=\omega_{p m}=2 \pi \nu \times \sqrt{2.55}$, (c) emerging Airy beam distribution, DNM's $\omega_{p e}=\omega_{p m}=2 \pi \nu \times \sqrt{3.1}$, and (d) emerging Airy beam distribution, DNM's $\omega_{p e}=\omega_{p m}=2 \pi \nu \times \sqrt{2}$.

As we all known, the nondiffracting beams could be extended to infinite space and have infinite energy; they are generally unobtainable in the laboratory. However, from the above discussion, we can come to the conclusion that the selfrecovery and self-reproducing Airy beam can be achieved in long distance by using periodic slab system with DNMs as long as $n_{l}=-n_{r}, L=Z$; the result is very important to form stable and excellent beam quality. When $n_{l} \neq-n_{r}$, the larger $n_{l}$ requires smaller $L$ to just compensate self-bending in RHMs; otherwise, the conclusion is also the opposite. $L \neq Z$ manifests that the slab system is quasiperiodic, which increases the difficulty of materials production process. Moreover, the quantitative relation between $L$ and $n_{l}$ of these quasiperiodic slab systems remains to explore and perfect further.

In practice, losses in DNMs are inevitable; in this subsection, emerging intensity profiles with four different collision frequencies $\gamma_{e}$ are displayed in Figure 4. It is clearly acquired that the larger the collision frequencies $\gamma_{e}$ are, the more serious the Airy beam deformation are formed. When $\gamma_{e}=$ $20 \mathrm{THz}$, only main lobe of Airy beam existed; other side lobes are vanished or significantly weakened from Figure 4(d), which indicates that the beam is seriously distorted. Similarly, side views of Airy beam propagating in each RHM and DNM unit successively changing with two kinds of collision frequencies $\gamma_{e}$ are exhibited in Figure 5. We can easily find that the beam propagation is rarely affected when $\gamma_{e}=0.2 \mathrm{THz}$ from Figure 5(a); however, as $\gamma_{e}$ gradually increases, the beam's attenuation and diffraction become more and more obvious; when $\gamma_{e}=20 \mathrm{THz}$, the interaction and diffraction between main lobe and side lobes lead to a distorted Airy beam; at this point, the bit error rate of communication system would greatly increase as to optical encryption. GrisSánchez et al. have designed and made a periodic optical fiber that guides an approximate Airy pattern as one of 


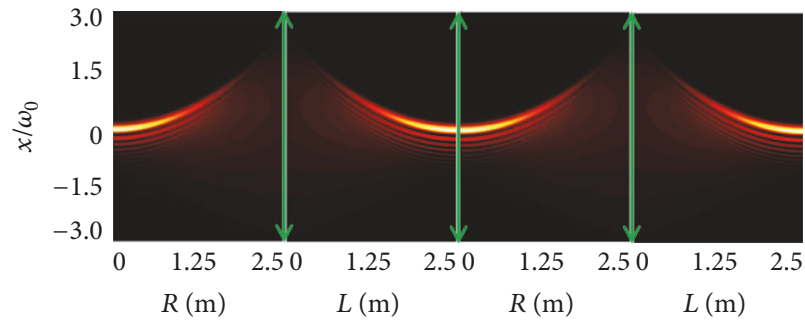

(a)

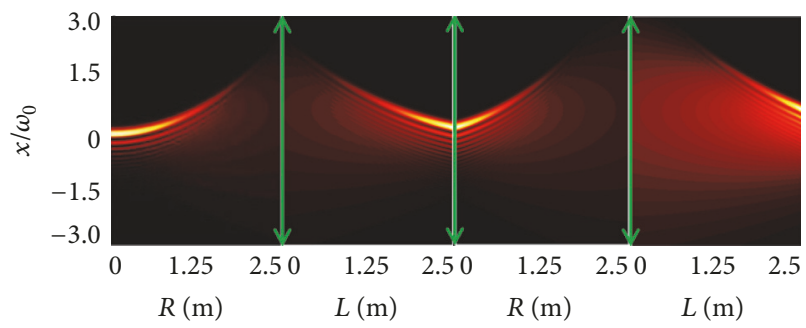

(c)

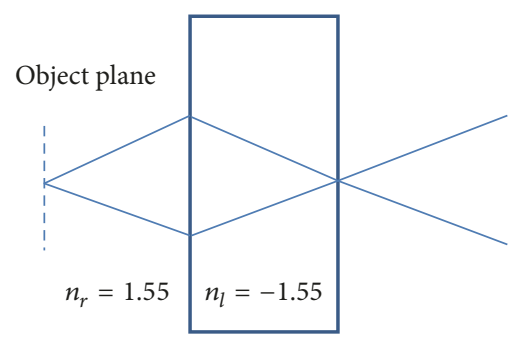

(b)

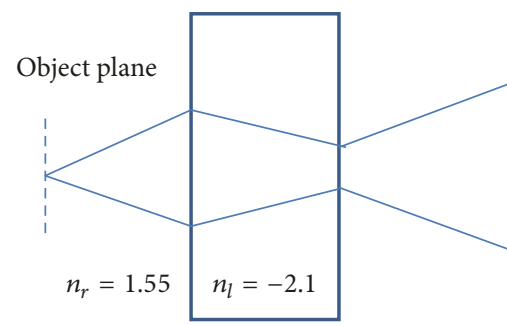

(d)
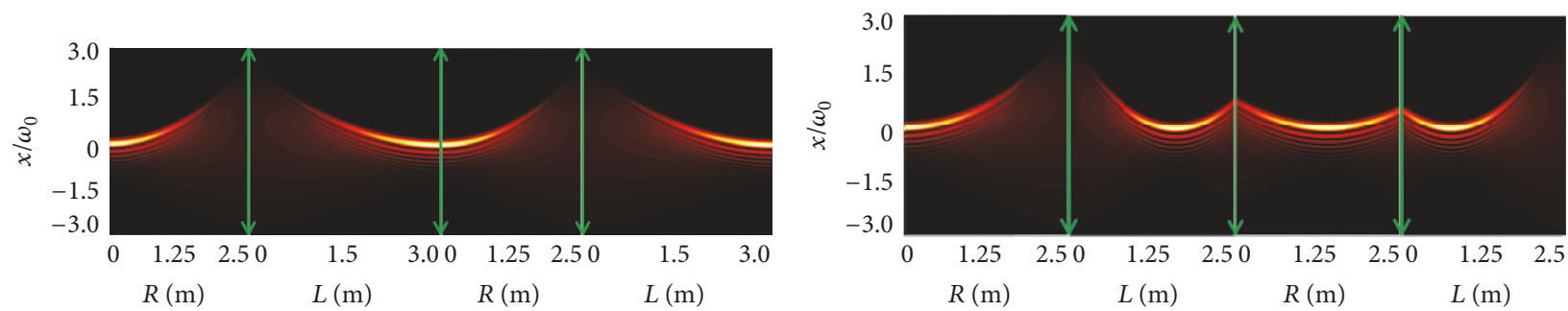

(e)

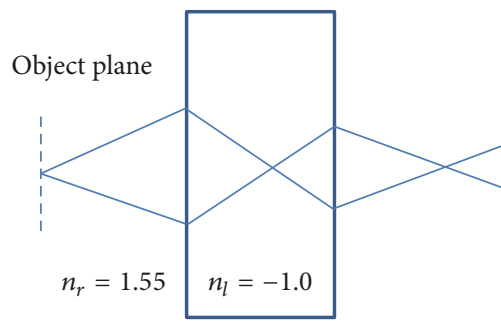

(g)

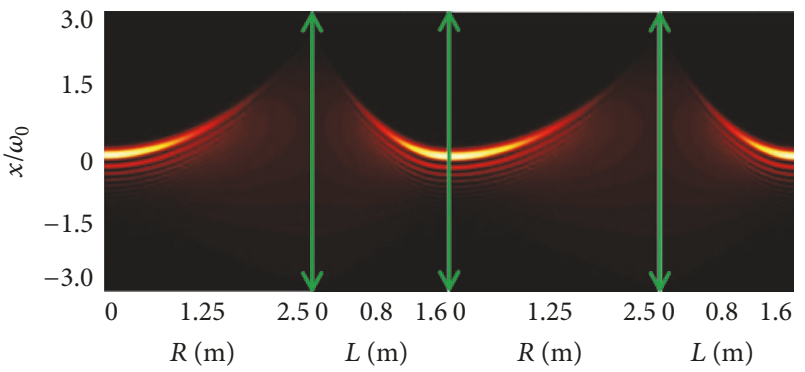

(h)

FIGURE 3: Side view of Airy beam propagating in each RHM and DNM unit successively with three kinds of DNMs and corresponding principle diagrams. (a) DNM's $\omega_{p e}=\omega_{p m}=2 \pi \nu \times \sqrt{2.55}$, (b) principle diagram where beam can just be compensated by each DNM, (c) DNM's $\omega_{p e}=\omega_{p m}=2 \pi \nu \times \sqrt{3.1}$, (d) principle diagram where beam cannot be completely compensated by each DNM, (e) Airy beam transmission in quasiperiodic slab system, $Z=2.5, L=105 / 31$, (f) DNM's $\omega_{p e}=\omega_{p m}=2 \pi v \times \sqrt{2}$, (g) principle diagram where beam is overcompensated by each DNM, and (h) Airy beam transmission in quasiperiodic slab system, $Z=2.5, L=50 / 31$.

the beam guided modes recently; the fiber's attenuation was $11.0 \mathrm{~dB} / \mathrm{km}$ at $1.55 \mu \mathrm{m}$ wavelength [24]. Here we estimate maximum collision frequencies value allowed by using their experimental result. Beam loss equation can be defined as [25]

$$
\alpha(\lambda)=-\frac{10}{(Z+L)^{2}} \lg \frac{I_{\text {out }}}{I_{\text {in }}},
$$

where $\alpha(\lambda)$ is loss coefficient, $I_{\text {in }} ; I_{\text {out }}$ denote incident beam intensity and emerging beam intensity, respectively. By substituting $Z=L=2.5 \mathrm{~m}$ and $\alpha(\lambda)=11.0 \mathrm{~dB} / \mathrm{km}$ into (13), we obtain $I_{\text {out }} / I_{\text {in }}=0.975$. When DNM's collision frequencies $\gamma_{e}$ and $\gamma_{m}$ equal $0.2 \mathrm{THz}$, the corresponding $I_{\text {out }} / I_{\text {in }}=0.980 \approx$ 0.975 ; hence, we had better choose $\gamma_{e}$ and $\gamma_{m}$ no more than $0.2 \mathrm{THz}(0.1 \%$ of the frequency $v)$ in order to achieve accurate transmission of optical communications.

\section{Conclusion}

In summary, we have studied the evolution of Airy beam propagating in periodic slab system with different negative 


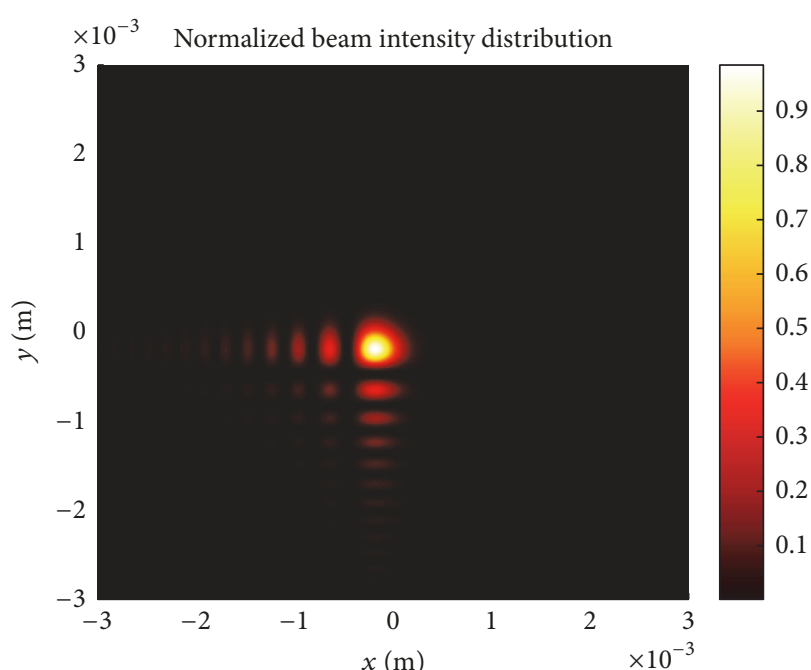

(a)

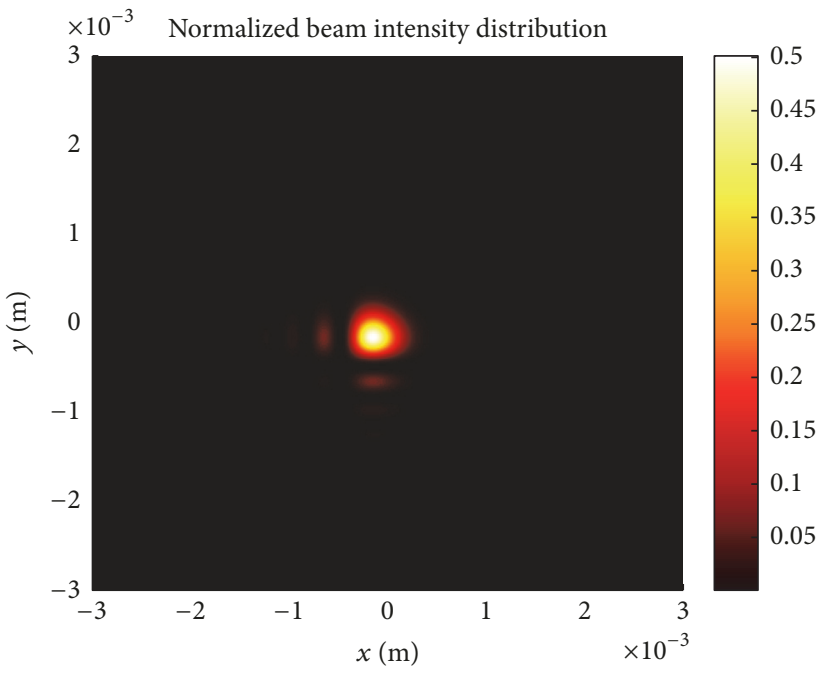

(c)

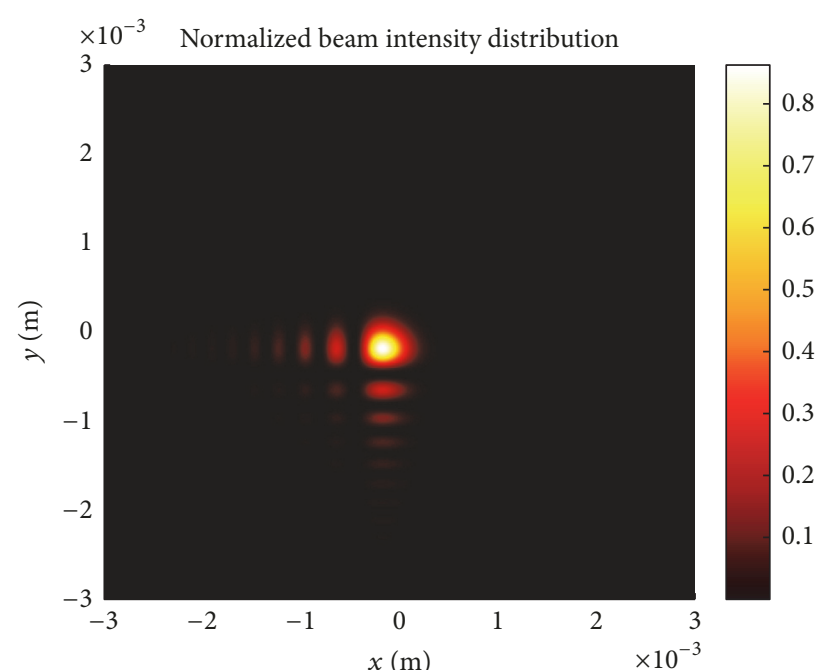

(b)

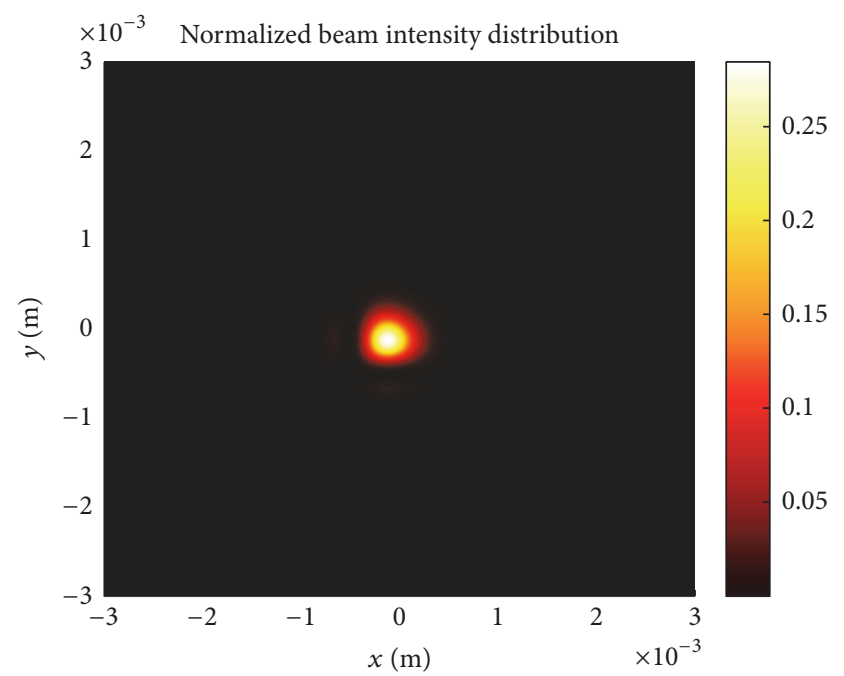

(d)

FIGURE 4: Emerging beam intensity profiles with four different collision frequencies $\gamma_{e}$. (a) $\gamma_{e}=0.2 \mathrm{THz}$, (b) $\gamma_{e}=2.0 \mathrm{THz}$, (c) $\gamma_{e}=10 \mathrm{THz}$, and (d) $\gamma_{e}=20 \mathrm{THz}$.

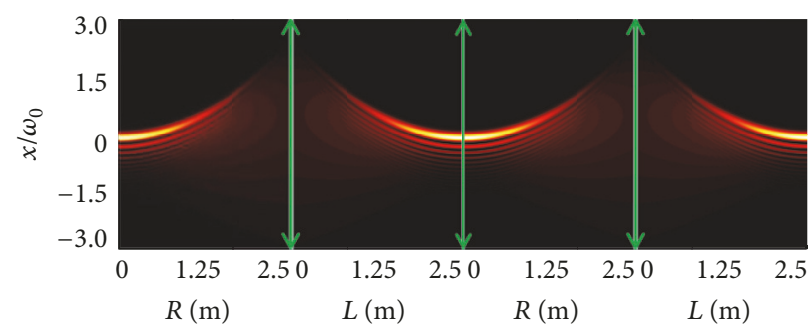

(a)

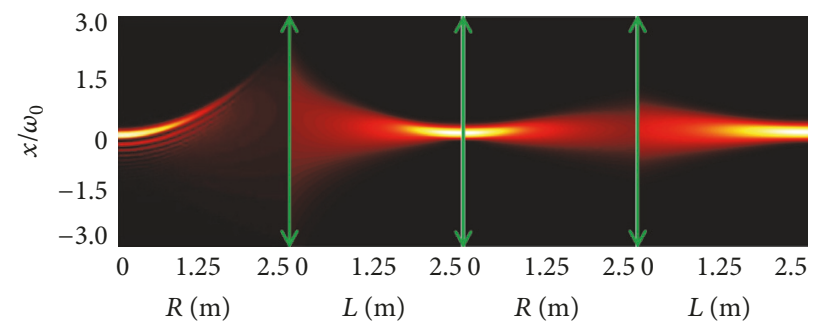

(b)

FIGURE 5: Side view of Airy beam propagating in each RHM and DNM unit changing with two different collision frequencies $\gamma_{e}$. (a) $\gamma_{e}=$ $0.2 \mathrm{THz}$; (b) $\gamma_{e}=20 \mathrm{THz}$.

index materials and its transmission mechanism. The emerging intensity profiles on output surface of slab system and side view of Airy beam transmission in each RHM and DNM unit including lossless and losses DNMs have been discussed. It is revealed that the self-recovery Airy beam can be achieved in long distance by using lossless periodic slab system with negative index materials as long as $n_{l}=-n_{r}, L=Z$; the conclusion is very important to forming stable and excellent 
beam quality. As to losses periodic slab system contained DNMs, the larger the collision frequencies $\gamma_{e}$ and $\gamma_{e}$ are, the more serious the Airy beam deformation is formed; hence, we had better choose $\gamma_{e}$ and $\gamma_{m}$ no more than $0.1 \%$ of the frequency $v$ in order to achieve accurate transmission of optical communications. It is expected that the proposed manner of wave transmission and the corresponding conclusions can be useful for the extension applications of optical control, especially for optical communication and optical encryption technique.

\section{Conflicts of Interest}

The authors declare that there are no conflicts of interest regarding the publication of this paper.

\section{Acknowledgments}

The authors gratefully acknowledge the financial support from Hubei Provincial Department of Education in China (no. B2017085).

\section{References}

[1] M. V. Berry and N. L. Balazs, "Nonspreading wave packets," American Journal of Physics, vol. 47, no. 3, pp. 264-267, 1979.

[2] G. A. Siviloglou, J. Broky, A. Dogariu, and D. N. Christodoulides, "Observation of accelerating airy beams," Physical Review Letters, vol. 99, no. 21, Article ID 213901, 2007.

[3] Y. Hu, G. A. Siviloglou, P. Zhang, N. K. Efremidis, D. N. Christodoulides, and Z. Chen, "Self-accelerating airy beams: Generation, control, and applications," Springer Series in Optical Sciences, vol. 170, pp. 1-46, 2012.

[4] J. Baumgartl, M. Mazilu, and K. Dholakia, "Optically mediated particle clearing using Airy wavepackets," Nature Photonics, vol. 2, no. 11, pp. 675-678, 2008.

[5] G. Porat, I. Dolev, and O. Barlev, "Airy beam laser," Optics Letters, vol. 36, no. 20, pp. 4119-4121, 2011.

[6] D. Abdollahpour, S. Suntsov, D. G. Papazoglou, and S. Tzortzakis, "Spatiotemporal Airy light bullets in the linear and nonlinear regimes," Physical Review Letters, vol. 105, no. 25, Article ID 253901, 2010.

[7] A. Minovich, A. E. Klein, N. Janunts, T. Pertsch, D. N. Neshev, and Y. S. Kivshar, "Generation and near-field imaging of airy surface plasmons," Physical Review Letters, vol. 107, no. 11, Article ID 116802, 2011.

[8] P. Polynkin, M. Kolesik, J. V. Moloney, G. A. Siviloglou, and D. N. Christodoulides, "Curved plasma channel generation using ultraintense airy beams," Science, vol. 324, no. 5924, pp. 229-232, 2009.

[9] N. Voloch-Bloch, Y. Lereah, Y. Lilach, A. Gover, and A. Arie, "Generation of electron Airy beams," Nature, vol. 494, no. 7437, pp. 331-335, 2013.

[10] H. A. Macleod, “Thin-Film Optical Filters,” CRC press, 2001.

[11] E. Ritter, "Properties of optical film materials," Applied Optics, vol. 20, no. 1, pp. 21-25, 1981.

[12] D. R. Smith, J. B. Pendry, and M. C. K. Wiltshire, "Metamaterials and negative refractive index," Science, vol. 305, no. 5685, pp. 788-792, 2004.
[13] V. G. Veselago, "The electrodynamics of substances with simultaneously negative values of $\varepsilon$ and $\mu$," Soviet Physics Uspekhi, vol. 10, no. 4, pp. 509-514, 1968.

[14] A. Alù and N. Engheta, "Pairing an Epsilon-Negative Slab with a Mu-Negative Slab: Resonance, Tunneling and Transparency," IEEE Transactions on Antennas and Propagation, vol. 51, no. 10 I, pp. 2558-2571, 2003.

[15] Y.-T. Fang, J. Zhou, and E. Y. B. Pun, "High-Q filters based on one-dimensional photonic crystals using epsilon-negative materials," Applied Physics B: Lasers and Optics, vol. 86, no. 4, pp. 587-591, 2007.

[16] W. T. Lu, J. B. Sokoloff, and S. Sridhar, "Refraction of electromagnetic energy for wave packets incident on a negative-index medium is always negative," Physical Review E, vol. 69, no. 2, 26604 pages, 2004.

[17] J. Chen, Y. Wang, B. Jia et al., "Observation of the inverse Doppler effect in negative-index materials at optical frequencies," Nature Photonics, vol. 5, no. 4, pp. 239-242, 2011.

[18] A. Mizrahi and Y. Fainman, "Negative radiation pressure on gain medium structures," Optics Letters, vol. 35, no. 20, pp. 3405-3407, 2010.

[19] M. W. Feise and Y. S. Kivshar, "Sub-wavelength imaging with a left-handed material flat lens," Physics Letters, Section A: General, Atomic and Solid State Physics, vol. 334, no. 4, pp. 326330, 2005.

[20] G. A. Siviloglou and D. N. Christodoulides, "Accelerating finite energy Airy beams,” Optics Letters, vol. 32, no. 8, pp. 979-981, 2007.

[21] K. Cheng, J.-S. Xia, and X.-Q. Zhong, "Propagation dynamics and vortex trajectory of an Airy vortex beam in gradient-index media," Acta Photonica Sinica, vol. 43, no. 9, Article ID 0905002, 2014 (Chinese).

[22] H.-C. Lin and J.-X. Pu, "Propagation of Airy beams from righthanded material to left-handed material," Chinese Physics B, vol. 21, no. 5, Article ID 054201, 2012.

[23] J. B. Pendry, "Negative refraction makes a perfect lens," Physical Review Letters, vol. 85, no. 18, pp. 3966-3969, 2000.

[24] I. Gris-Sánchez, D. Van Ras, and T. A. Birks, “The airy fiber: An optical fiber that guides light diffracted by a circular aperture," Optica, vol. 3, no. 3, Article ID 030270, pp. 270-276, 2016.

[25] G. P. Agrawal, Nonlinear Fiber Optics, Academic Press, 2007. 

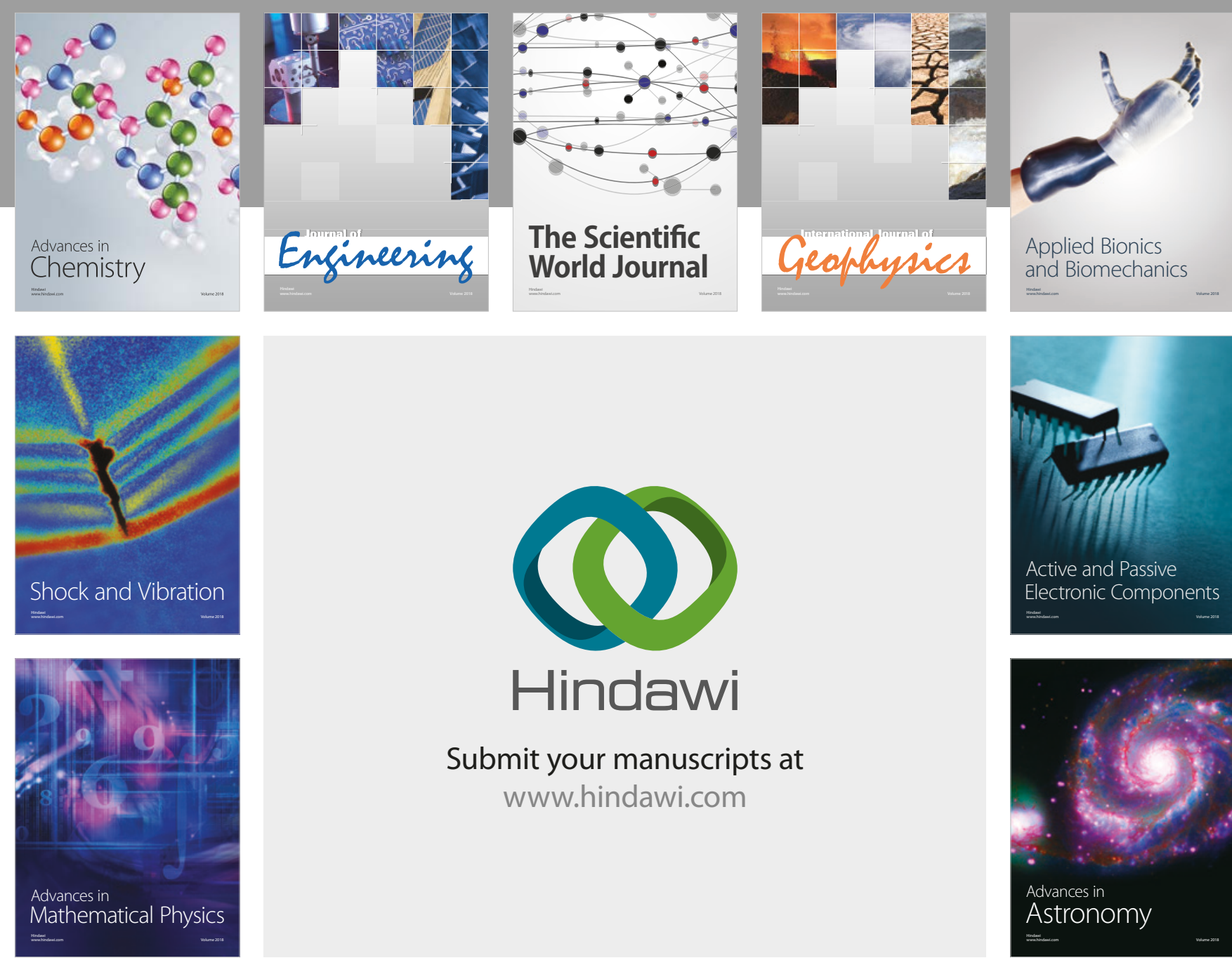

Submit your manuscripts at

www.hindawi.com

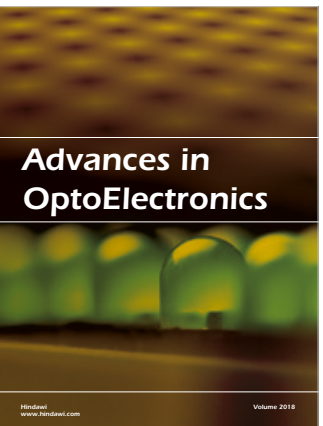

\section{Rotcting Machinery}
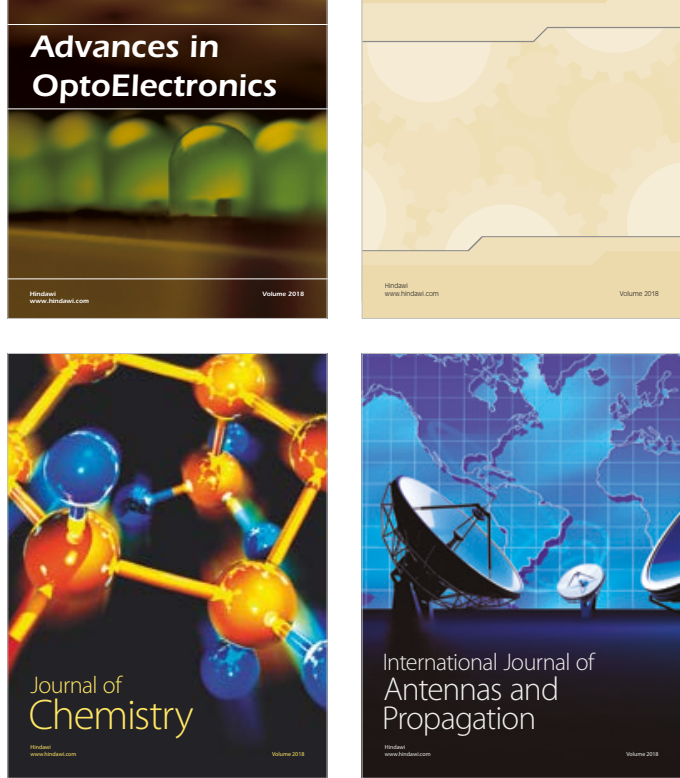

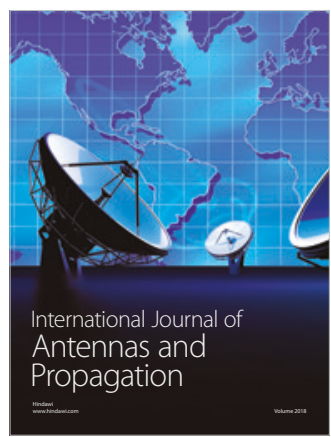

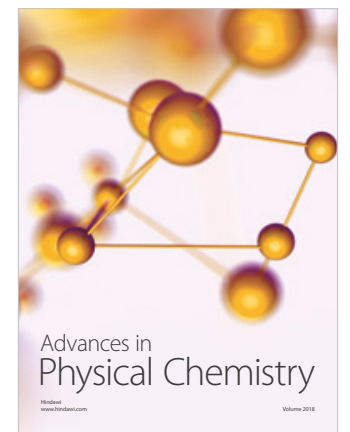

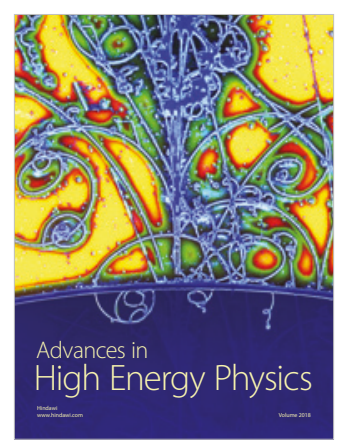

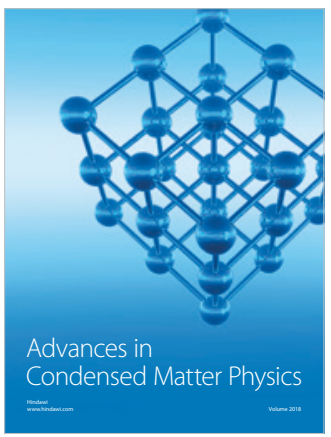

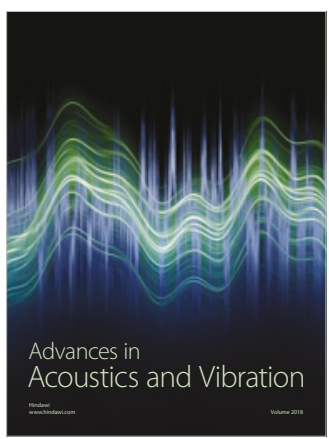

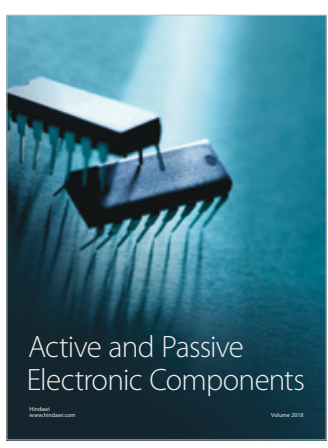
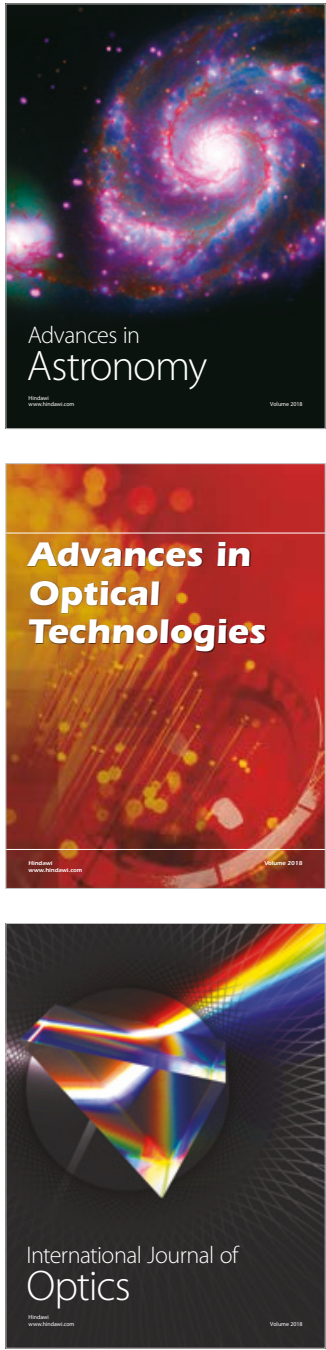